\title{
IDEE E CONOSCENZA NELLE POLITICHE PUBBLICHE EUROPEE: \\ TECNOCRAZIA O POLITICIZZAZIONE?
}

\author{
di Claudio M. Radaelli
}

La conoscenza nelle politiche pubbliche dell'Unione europea: un vantaggio a metà?

Come spesso accade, l'interesse attuale per la dimensione cognitiva della politica rappresenta più una riscoperta che una novità assoluta. In senso lato, essa comprende filoni ben noti, addirittura classici, degli studi politici. Lo dimostra la ricca letteratura su temi diversi quali l'incrementalismo (Lindblom 1959), la razionalità limitata (Simon 1957), le mappe cognitive delle élites politiche (Axelrod 1976), la concezione dei meccanismi di governo come apprendimento (Deutsch 1966, 80; Heclo 1972), il cosiddetto «complesso militare-industriale» (Rosen 1973), e la tecnocrazia (Fisichella 1997). Perché dunque «riscoprire» il ruolo politico della conoscenza? $\mathrm{O}$ meglio, qual è il potenziale di studi i quali, sia nell'ambito dell'analisi sulle politiche pubbliche (Capano 1995; Radaelli 1997) sia nelle relazioni internazionali (Jacobsen 1995), reclamano una maggiore considerazione per i fattori cognitivi nelle scelte di policy? Quanto sono efficaci nella spiegazione del cambiamento (policy change)? $\mathrm{E}$, da ultimo, come possono essere valutati da un punto di vista normativo? Una delle idee cardine della prospettiva cognitiva è che la politica vada oltre la semplice risoluzione dei conflitti. L'apprendimento, la propensione alla soluzione dei problemi, i fora di discussione e il policy enlightenment - si sostiene - possono contare più della politica basata su stili conflittuali. La po-

Desidero ringraziare la Nuffield Foundation (grant SOC/100/001577) e - senza implicarli in quanto scritto - $i$ partecipanti ai seminari in cui bo presentato una versione preliminare di questo saggio (all'Iue il 27 aprile 1999; al Workshop «The state of the art: theoretical approaches to the European Union in the post-Amsterdam era», Aston University, 6-7 maggio 1999).

RIVISTA ITALIANA DI SCIENZA POLITICA / a. XXIX, n. 3, dicembre 1999 
litica sta forse superando il modello della democrazia competitiva-avversariale?

Tali questioni hanno una particolare importanza nello studio delle politiche pubbliche dell'Unione europea (Ue): il ruolo dell'expertise in questo settore è divenuto oggetto di interesse accademico e di appassionato dibattito. Diversi studi hanno chiarito come la conoscenza intervenga nella formazione delle politiche pubbliche in virtù della presenza di esperti e comunità epistemiche (Richardson 1996; Zito 1998), di fora di discussione (Coen 1997), e dell'imprenditorialità della Commissione europea (Cram 1993), un'organizzazione che ha come sua principale risorsa proprio la conoscenza. Ciò è di buon auspicio in termini di qualità del processo democratico. Stiamo allora andando verso scelte politiche più informate, forse persino illuminate?

La dimensione cognitiva della politica ha tuttavia anche un lato oscuro: il suo nome è tecnocrazia. Non sorprenderà, a questo proposito, che politiche gestite da esperti e imprenditori di policy non elettivi finiscano sotto accusa per prime. Mentre la democrazia è basata su consenso, libere elezioni e partecipazione, la tecnocrazia attribuisce all'expertise il ruolo di unico fondamento dell'autorità e del potere. La questione viene sollevata con accenti diversi dalla stampa - emblematiche le reazioni alle dimissioni della Commissione Santer nel marzo 1999 - dagli attori politici, e dagli studi di tipo teorico-normativo sul deficit democratico dell'Unione (O'Neill 1999; Wincott 1998).

Che cosa si intende, però, con tecnocrazia nel caso dell'Ue? Come possono gli studiosi dell'Unione europea essere allo stesso tempo affascinati dall'ipotesi di una politica post-conflittuale e inorriditi da un processo di elaborazione delle scelte di tipo tecnocratico?

Lo scopo di questo saggio è triplice. Innanzitutto, spiegherò in che misura termini come tecnocrazia, stato regolatore (regulatory state), politica burocratica e comunità epistemiche gettano luce su diversi aspetti della politica della conoscenza nell'Unione europea. Il secondo obiettivo è offrire un quadro ragionato dei diversi filoni della letteratura che puntano verso una «prospettiva cognitiva» sulle politiche pubbliche comunitarie. Sebbene questo studio sia limitato all'Ue, alcune osservazioni fanno riferimento a più ampi dibattiti relativi all'analisi teorica delle politiche pubbliche. Da ultimo, formulerò le mie riflessioni sulle implicazioni normative dell'analisi. Il secondo para- 
grafo introduce il tema della politica dell'expertise nelle scelte comunitarie attraverso la chiarificazione di alcuni concetti: quelli di tecnocrazia, politica burocratica e comunità epistemiche. Questi tre concetti descrivono diverse forme di expertise che operano nel processo di formulazione delle politiche pubbliche, e questa differenza richiede di essere spiegata. Il terzo paragrafo valuta il potenziale euristico dell'approccio fondato sulla conoscenza in rapporto alle politiche pubbliche dell'Ue, rivolgendo una particolare attenzione al mutamento politico. Infine, il quarto paragrafo riflette - in via sostanzialmente sperimentale su questioni di carattere normativo.

Tecnocrazia, politica burocratica e comunità epistemiche: in che modo conta l'expertise?

Dal momento della problematica ratifica del Trattato di Maastricht, il dibattito sulla natura tecnocratica dell'Unione europea è andato intensificandosi, fino alle clamorose dimissioni della Commissione Santer nel marzo 1999. Ma che cosa significa tecnocrazia oggi? Una vasta letteratura ha discusso l'evoluzione della tecnocrazia come concetto e come ideologia politica (Burris 1993; Fischer 1990; Fisichella 1997; Sartori 1987; Williams 1971). Tale letteratura ha anche sottolineato la principale differenza tra le prime utopie tecnocratiche e il tecnocrate di oggi. Se in origine veniva auspicata l'assunzione diretta del potere da parte degli esperti, la tecnocrazia contemporanea rispetta formalmente $i$ valori e le istituzioni democratiche. Nessuno oggigiorno si dichiara a favore di un governo di scienziati, né si sostiene che le istituzioni formali della democrazia debbano essere trasformate in soviet di tecnocrati à la Veblen. Ma i tecnocrati (tanto nelle politiche nazionali quanto in quella europea) stanno guadagnando potere, all'ombra di strutture formali: organi consultivi (Boston 1988), think tanks (Stone et al. 1998), banche centrali $\mathrm{e}$ autorità regolative indipendenti (Majone 1996), congiure - per dirla con Wallis (1997) - di riformatori tecnocratici e infine governi affollati di ministri non eletti sono gli ambiti primari nei quali gli esperti hanno ormai guadagnato un ruolo centrale.

Il punto da sottolineare non riguarda tuttavia le persone (esperti al posto di politici eletti), quanto il cambiamento nella natura del potere (Fischer 1990). Molti dei cambiamenti degli 
ultimi vent'anni (come le privatizzazioni, la deregolazione, la riforma amministrativa e i nuovi paradigmi di politica monetaria) sono stati caratterizzati da una clamorosa battaglia delle idee. Allo stesso tempo, la sfera pubblica ha attraversato - sostiene Fischer - un processo di de-politicizzazione. A causa della loro complessità, le politiche pubbliche sono spesso isolate dal dibattito pubblico e dalle pressioni dal basso. Questa opacità delle politiche pubbliche è, di conseguenza, un tratto importante del mutamento del potere. I movimenti sociali, gli interessi diffusi e la pubblica opinione sono ridotti al silenzio in quanto privati della loro possibilità di esprimersi (voice) in materia di politiche pubbliche.

Il mutamento nella natura del potere è accompagnato dall'affermarsi di una mentalità tecnocratica fra le élites amministrative. Il tecnocrate è sostanzialmente ostile alla trasparenza della democrazia e sospettoso delle istituzioni parlamentari (Putnam 1977; vedi anche Meynaud 1969). Il conflitto politico non è considerato un ingrediente salutare della democrazia, ma una conseguenza dell'ignoranza. L'analisi razionale e la conoscenza - così recita il mantra tecnocratico - producono soluzioni efficienti che dovrebbero venire accettate da tutti gli individui di buona volontà. Di conseguenza «il tecnocrate ritiene che il conflitto sociale e politico sia spesso, nella migliore delle ipotesi, fuorviante, e nella peggiore artificioso» (Putnam 1977, 386). Un corollario è che la mentalità tecnocratica è più interessata al progresso tecnologico, all'efficienza e alla produttività materiale che all'equità e alla giustizia sociale (Putnam 1977, 387).

La concezione originaria dell'integrazione europea e la sua eredità custodiscono gelosamente numerose componenti tecnocratiche. La prima fase dell'integrazione europea è stata segnata da un approccio tecnocratico incarnato dal Piano Monnet per l'istituzione della Ceca (Featherstone 1994; Monnet 1978) ${ }^{1}$. L'idea chiave del progetto era costituita dalla speciale posizione attribuita agli esperti nella formulazione delle politiche pubbliche sovranazionali. Di qui l'idea di una Alta Autorità composta

1 Fulvio Attinà mi ha ricordato che questa fase iniziale è stata segnata anche da un'intensa attività intellettuale in favore della democratizzazione del processo integrativo. Tecnocrazia e democratizzazione erano dunque entrambe simultaneamente presenti nel dibattito, ma soltanto uno dei numerosi progetti per la realizzazione della Ceca e della Cee si è poi realizzato, e si trattava di quello tecnocratico. 
da esperti qualificati ${ }^{2}$. Questi esperti, tuttavia, non avrebbero dovuto operare in isolamento rispetto alla società. Il metodo Monnet per l'integrazione prefigurava un sistema di engrenage in virtù del quale reti di gruppi di interesse, lavoratori e imprese organizzati sarebbero stati gradualmente coinvolti nella formulazione delle scelte comunitarie (Haas 1958). I padri fondatori dell'integrazione europea avevano previsto che il meccanismo di governo (governance) consistesse nel consenso tecnocratico. Il motore dell'integrazione sarebbe stato rappresentato da una combinazione di tecnocrati e gruppi di interesse, che insieme avrebbero costruito coalizioni sovranazionali a sostegno della comunità (Haas 1958). Wallace e Smith $(1995,140)$ sottolineano come il cittadino qualunque fosse completamente trascurato da questo approccio: «La strategia di Monnet consisteva in un gradualismo trainato dall'élite, con l'aspettativa che il consenso popolare sarebbe cresciuto col tempo. In un simile approccio indiretto all'integrazione politica, il fatto di attirare le organizzazioni interessate - dell'impresa, del lavoro e degli enti amministrativi nazionali - era una priorità assai più sentita del coinvolgimento diretto di un pubblico all'epoca ancora poco informato». Monnet non aveva neppure pensato ad un Consiglio dei Ministri, probabilmente perché l'Alta Autorità aveva una competenza limitata, circoscritta alle politiche per l'acciaio e il carbone. Ma ciò è indicativo del suo approccio tecnocratico. Toccò alle delegazioni belga e olandese di sollevare la questione di un Consiglio che controbilanciasse l'Alta Autorità e fissasse la linea politica. Come ha mostrato Featherstone (1994, 159 e 161), la tendenza a trascurare la dimensione politica è sopravvissuta nel corso degli anni.

Questa eredità tecnocratica non riguarda soltanto la Commissione, ma permea tutta la struttura politica dell'Unione. Uno studio esauriente ha sostenuto che l'Ue è basata su tre meccanismi di rappresentanza (Andersen e Burns 1996, 230): la rappresentanza degli esperti ( in contesti nei quali contano in modo particolare "razionalità" ed efficacia» - chiariscono Andersen e Burns); la rappresentanza dei gruppi di interesse (e degli interessi diffusi) in reti di policy; e la rappresentanza nazionale (in virtù della costante presenza di rappresentanti degli stati mem-

2 Essendo quasi esclusivamente interessata alle politiche per il carbone e l'acciaio, l'Alta Autorità aveva un ambito di attività completamente diverso da quello della Commissione che conosciamo oggi. 
bri nelle scelte comunitarie). I primi due meccanismi indicano una significativa depoliticizzazione e opacità delle politiche pubbliche, un tratto caratteristico della tecnocrazia.

Andersen e Burns notano come il processo di formazione delle politiche pubbliche nell'ambito dell'Unione europea punti soprattutto all'efficienza. Tale enfasi dipende dal fatto che l'Unione è andata caratterizzandosi come sistema politico con un vantaggio comparato nella produzione di politiche regolati$\mathrm{ve}^{3}$. A questo proposito, l'Ue si è sviluppata in modo diverso dagli stati nazionali. Infatti non ne ha seguito, e probabilmente non ne seguirà, le traiettorie tipiche di sviluppo politico, così come le abbiamo ricostruite guardando all'Europa occidentale. Rispetto alla vicenda dello stato nazionale, non si registra soltanto una discontinuità in termini di sviluppo istituzionale (parlamento, governo e burocrazia cambiano radicalmente forma e sostanza quando si passa dal livello nazionale a quello dell'Unione), ma la specializzazione in termini di tipi di politiche pubbliche è assai diversa. Mentre gli stati nazionali si sono sviluppati e consolidati attorno a politiche distributive e redistributive (in sintesi, politiche di welfare e fiscali), l'Ue - come hanno dimostrato gli studi condotti da Gian Domenico Majone - ha sviluppato soprattutto la dimensione regolativa. I vincoli di bilancio dell'Unione sbarrano la strada all'attuazione di politiche distributive e redistributive (dato che i programmi di politica sociale richiedono grandi somme di denaro), ma per fissare una regola che, ad esempio, indichi i requisiti per operare nel settore del credito o per fusioni e acquisizioni internazionali, l'unica risorsa necessaria è una profonda conoscenza dei mercati e delle questioni che debbono essere regolamentate. La Commissione è, senza ombra di dubbio, una burocrazia nell'ambito della quale le risorse cognitive sono estremamente sviluppate.

Per quanto riguarda il rapporto con i soggetti regolati, la Commissione è al centro di dense reti di interazione, come ben illustrato dalla letteratura sui policy networks comunitari (Peterson 1992; Richardson 1995). Pur non trattando di questioni comunitarie, è interessante osservare come Giuliani (1998) combini la dimensione cognitiva con la costruzione strategica di reti nella sua disamina dell'imprenditorialità. Nei suoi scritti Majone

3 Tipicamente, la politica regolativa è una risposta ai fallimenti di mercato: la sua logica fondamentale è di ristabilire l'efficienza quando si determinano fallimenti di mercato. 
propone l'idea del copinage technocratique per indicare un intenso gioco di interazioni tra i funzionari di Bruxelles, quelli degli stati membri e gli esperti dell'industria. Il sistema originario costituito dall'engrenage, immaginato da Monnet, gioca ancora un ruolo estremamente significativo, seppure in forme diverse.

La Commissione, come tutte le burocrazie, tende inoltre ad ampliare i propri poteri. Il compito di promuovere e rafforzare l'integrazione europea è persino fissato dai Trattati, ed è proprio a ciò che ci si riferisce quando si parla della Commissione come del «motore dell'integrazione». Gli studiosi delle politiche pubbliche europee hanno messo in rilievo il ruolo della Commissione come imprenditore di policy, una formula che connota un soggetto capace di sfruttare opportunisticamente le risorse scarse a sua disposizione per creare nuove politiche (Cram 1993).

Va oltre gli obiettivi di questo lavoro valutare le implicazioni della politica della regolazione per la teoria dell'integrazione europea (vedi comunque Caporaso 1996). Sarà in questa sede sufficiente osservare che l'Ue è un sistema politico con un vantaggio comparato nella produzione di regolazione. La conoscenza, e non il bilancio, è la risorsa critica nella produzione di politiche di regolazione, e la Commissione utilizza questa risorsa ampiamente. Le politiche regolative hanno come obiettivo l'efficienza, piuttosto che la redistribuzione. Ciò le rende adatte ad essere discusse e negoziate da esperti, mentre questioni di carattere redistributivo suscitano la passione dei politici, dei partiti e dell'opinione pubblica a causa del loro impatto sulla struttura di classe. L'efficienza è un gioco a somma positiva, nel quale nessuno è svantaggiato. La redistribuzione, invece, implica che risorse siano sottratte ad una parte della società e attribuite ad un'altra. Questa insistenza sull'efficienza e sui giochi a somma positiva è coerente con l'elemento dominante della mentalità tecnocratica. Il tecnocrate ritiene che l'analisi razionale e la valutazione scientifica dei dati di fatto determinerà il raggiungimento di un consenso unanime nelle scelte pubbliche. Al contrario, il tecnocrate si sente a disagio in presenza di conflitto politico, dibattito ideologico, e controversie su questioni di giustizia sociale. Concludendo su questo punto, la politica della regolazione in ambito europeo è coerente con l'eredità tecnocratica del modello originario. Nella sua forma attuale, la regolazione è il tipo di politica che meglio si adatta alle risorse cognitive, alla tendenza al negoziato di tipo tecnico e all'inclinazione generale ad evitare il dibattito politico aperto. 
I semi della tecnocrazia hanno trovato nella politica della regolazione terreno fertile. Ma come vengono elaborate le politiche nel lavorio quotidiano del processo europeo? Il concetto di politica burocratica offre ulteriori spunti alla nostra analisi della politica dell'expertise nell'Ue (Allison 1971; Downs 1967 e per l'Ue Peters 1992). La politica burocratica, nell'ambito dell'Unione europea, assume due forme: la competizione burocratica fra (e addirittura all'interno delle) istituzioni e l'organizzazione delle politiche pubbliche intorno ad aree di policy funzionali. La competizione burocratica coinvolge istituzioni europee quali la Commissione, il Consiglio e il Parlamento. Nell'ambito di questa dinamica, la Commissione cercherà, ad esempio, di ampliare il proprio potere a danno di altre istituzioni europee. La concorrenza per l'acquisizione di maggiore potere non mette soltanto un'istituzione europea contro l'altra, ma è possibile che battaglie per la conquista di posizioni vengano combattute anche all'interno della medesima istituzione. Studi dedicati alla Commissione mostrano un'immagine delle Direzioni generali (Dg) con diverse culture amministrative (Cini 1996) e diversi policy frameworks (Harcourt 1998).

Il secondo livello della politica burocratica è quello delle singole aree di policy. L'attività di definizione delle politiche pubbliche è qui organizzata attorno a un certo numero di aree funzionali dove le Direzioni della Commissione, i Commissari, i comitati del Parlamento Europeo, i gruppi consultivi del Consiglio, gli amministratori nazionali e i gruppi di interesse formano coalizioni in competizione per l'acquisizione di potere (Peters 1992, 116; Richardson 1996). Frammentazione, formazione di coalizioni, bargaining, creazione di reti ed intensi negoziati in arene settoriali sono le caratteristiche di questo livello della politica burocratica.

Andrebbe poi aggiunto che il sistema politico europeo offre molteplici punti di accesso (Peters 1994). Non esiste un attore centrale che svolga funzioni di coordinamento, come un governo centrale. Non esistono maggioranze che, dopo le elezioni, diano vita a un governo con un mandato legislativo da adempiere. E il ruolo dei partiti politici nel controllare l'agenda pubblica è straordinariamente limitato se paragonato al loro ruolo nei sistemi politici interni. In questo senso, il processo di formazione delle politiche pubbliche dell'Unione può essere descritto come decentralizzato. Come spiega Peters, «che la burocrazia, europea o nazionale, sia o meno l'attore dominante (e spesso lo è), il 
processo di formazione delle politiche pubbliche ha le caratteristiche della decentralizzazione e del controllo locale tipici della forma burocratica del medesimo» (Peters 1992, 118).

La prevalenza della politica frammentata-settoriale ha conseguenze significative. Una è che il processo di scelta pubblica viene sottratto al controllo di tipo macro-politico e alla sorveglianza del pubblico. Un'altra è l'intensa competizione per il potere, il che spinge in senso opposto rispetto all'apprendimento e all'acquisizione di stili di soluzione dei problemi più cooperativi. Un processo «isolato», settoriale e tecnico costituisce una precondizione particolarmente favorevole al tipo di scelte pubbliche «a-politiche» descritto dalla letteratura tecnocratica.

In ogni caso, in determinate circostanze la principale questione in gioco non è la competizione fra istituzioni «che lottano per conservare il proprio potere», come scrive Peters. Le politiche dell'Unione spesso toccano aree caratterizzate da grande incertezza. Certe scelte ambientali o la manipolazione genetica sono esempi in cui l'elemento dominante è un'incertezza radicale. In queste condizioni il processo di definizione delle politiche pubbliche è anche puzzling, e non semplice powering (Heclo 1974). Questa considerazione richiama la nostra attenzione sul ruolo politico delle comunità di esperti.

A fronte del ruolo sempre più significativo degli argomenti scientifici e della incertezza radicale, un filone della letteratura nato di recente ha individuato la presenza di «comunità epistemiche» all'interno dell'Unione europea (Radaelli 1997; Richardson 1996; Verdun 1999; Zito 1998). Peter Haas ha elaborato il concetto di «comunità epistemica» proprio per analizzare il coordinamento delle politiche a livello internazionale. Il concetto ha l'obiettivo di «volgere lo studio del processo politico nell'interrogativo circa chi apprende che cosa, quando, a vantaggio di chi e perché» (Adler e Haas 1992, 370). L'enfasi dovrebbe essere posta sull'apprendimento perché, in condizioni di incertezza radicale, la conoscenza diventa un meccanismo fondamentale di sviluppo delle politiche pubbliche.

Il termine comunità epistemica definisce «una rete di professionisti con una expertise ed una competenza riconosciute in una particolare sfera e che autorevolmente vantano di possedere conoscenze di rilevanza politica» nell'ambito di quella sfera o area tematica (Haas 1992, 3). L'approccio basato sulle comunità epistemiche introduce tre dimensioni: l'incertezza, l'interpretazione e l'istituzionalizzazione delle idee. Dove vi è incertezza 
- sostiene Haas - non vi è una chiara percezione da parte dell'attore di quale sia il suo stesso interesse: una comunità epistemica, dal canto suo, è in grado di condurre ad una definizione degli interessi chiarendo determinate dimensioni di una questione, chiarimento dal quale ciascun attore può dedurre i suoi interessi. Gli interessi di conseguenza diventano una variabile dipendente dinamica, modellata dalla conoscenza. In questo modo - scrive Haas - «molte delle condizioni che inducono a concentrarsi sul potere sono assenti. E difficile per i leader identificare $\mathrm{i}$ loro potenziali alleati politici. Non saranno disponibili indicazioni tratte da considerazioni in termini di potere né di carattere istituzionale per definire il proprio comportamento, e possono così emergere nuovi stili d'azione» $(1992,14)$. L'incertezza comporta che gli attori si interroghino sulle politiche pubbliche. Coloro che suggeriscono un'interpretazione sono in una posizione chiave dato che la politica è innanzitutto e più di ogni altra cosa un tentativo di comprendere e decodificare una realtà complessa. Interessante, a questo proposito, è la definizione che Pierre Muller ha dato delle politiche pubbliche come «costruzione di un rapporto con il mondo», sottolineando l'analogia sotto il profilo politico della prise de parole (produzione di significato) e della prise de pouvoir, o strutturazione dei rapporti di potere (Muller 1995, 164). Detto in modo diverso, la produzione di significato è la chiave per la definizione degli interessi e l'istituzionalizzazione delle idee di policy.

Le comunità epistemiche hanno potere, tuttavia, soltanto quando sono inserite organicamente nel processo politico. Il loro ruolo è sottoposto a vincoli: spesso le comunità epistemiche «creano la realtà, ma non come vorrebbero» (Adler e Haas 1992, 381). Haas (1992) sostiene che le idee di una comunità epistemica influenzano le politiche pubbliche quando gli esperti raggiungono posizioni di potere burocratico, ad esempio attraverso l'istituzionalizzazione di comitati consultivi che debbono essere interpellati prima di assumere una determinata decisione. Nel valutare l'approccio basato sulle comunità epistemiche in relazione all'Unione europea, va osservato che il loro potere «è limitato dalla necessità dei soggetti chiamati a definire le politiche pubbliche - ai livelli comunitario e nazionale - di coinvolgere altri tipi di attori, in particolare i gruppi di interesse tradizionali» (Richardson 1996, 16). Più in generale, ci sono ottime ragioni per considerare i membri di una data comunità epistemica un attore (non l'unico, e spesso neppure il più potente) facente 
parte di coalizioni complesse in concorrenza in aree funzionali. Naturalmente, tutto dipende dal caso empirico in esame. $\mathrm{Ma}$, ad esempio, potrebbe darsi che in un'area di policy vi siano due coalizioni in concorrenza ed una coalizione sia assistita da una comunità epistemica sottostante (Radaelli 1997). La ragione teorica che induce a considerare l'interazione delle comunità epistemiche con coalizioni più ampie (Sabatier 1998) è che conoscen$\mathrm{za}$ e interessi intrattengono una relazione simbiotica.

In questo paragrafo sono stati introdotti termini quali tecnocrazia, politica della regolazione, politica burocratica e comunità epistemiche, tutti connessi alla crescente importanza della politica dell'expertise. Connessione non significa, tuttavia, identificazione. Non si dovrebbero infatti fondere tutti questi elementi nel concetto di tecnocrazia: non è consigliabile ampliare il concetto di tecnocrazia al punto di farvi rientrare comunità epistemiche, politica della regolazione e politica burocratica. Per questa ragione, procederemo verificando innanzitutto l'utilità del concetto di tecnocrazia e, quindi, contestualizzeremo le diverse manifestazioni della politica dell'expertise, contrastandole con i processi di politicizzazione.

Cominciamo dal concetto di tecnocrazia. In quanto concetto generico, quello di tecnocrazia è un termine ampio che s'accorda piuttosto bene con molti aspetti dell'Ue. Ma ad un'analisi più attenta non si dimostra molto utile. Tecnocrazia significa stato interventista, pianificazione, razionalizzazione delle politiche pubbliche e stabilizzazione dell'economia. L'Unione però può essere qualsiasi cosa, ma non interventista (il suo bilancio fluttua al di sotto dell'1,5\% del Pil europeo). Inoltre, il processo di formazione delle politiche pubbliche dell'Unione è giudicato molto instabile (Peters 1994; Richardson 1996).

Un altro punto critico è che concetti come tecnostruttura (Galbraith 1967), tecnocorporativismo (Fischer 1990), complesso militare-industriale (Rosen 1973) o tecnocomplesso (Williams 1971) non riguardano gli stessi attori che giocano un ruolo chiave nell'ambito dell'Ue. In altri termini, il referente sociologico di applicazione della logica tecnocratica non è facilmente rapportabile all'Unione. Il processo di formazione delle politiche pubbliche in seno all'Ue è frammentato e settorializzato: non esiste nulla che si avvicini ad un blocco di potere monolitico che governi questo processo.

Rivolgendo ora l'attenzione alle arene del potere, il processo di scelta comunitaria si articola in una serie di arene diverse. 
Studi dedicati alla formulazione dell'agenda in seno all'Ue hanno mostrato diversi punti di accesso per le domande politiche. La Commissione, il Parlamento, la Corte di Giustizia, ma anche le istituzioni politiche nazionali sono arene di primaria importanza per la politica dell'Unione. Tanto che gli attori si impegnano in quello che è stato definito «venue-shopping» (Richardson 1996; Sabatier 1998) e si rivolgono all'arena dove possono sfruttare meglio le loro risorse. Il problema che sorge a questo punto è come possa una classe tecnocratica controllare tutte queste arene. E infatti la pluralità delle arene costituisce un problema molto serio per la teoria tecnocratica ${ }^{4}$.

Per quanto riguarda l'ideologia, la tecnocrazia può proliferare in condizioni di sfiducia nei confronti dei politici. Perché la tecnocrazia possa avere successo, la decisone politica deve essere percepita come lenta, corrotta e, in ultima istanza, irrazionale. Ma la gente non si fida dei burocrati di Bruxelles. Se è possibile enucleare una domanda che proviene dall'opinione pubblica, è quella di una maggiore presenza della politica, non di un suo ridimensionamento.

La teoria tecnocratica, insomma, è più un punto di partenza che un punto di arrivo (Fisichella 1997). La figura $1^{5}$ si propone quindi di situare la politica tecnocratica dentro un più ampio schema teorico per l'analisi dell'expertise. L'obiettivo non è quello di sottolineare la presenza di attori diversi nelle politiche pubbliche, ma di indicare diverse logiche in modo ideal-tipico. Inoltre, la figura andrebbe letta in modo dinamico: lo stesso tema potrebbe infatti viaggiare lungo le due dimensioni della figura, per esempio acquistando visibilità politica. Il conflitto sulla definizione dei problemi riguarda spesso coloro che spingono per una definizione tecnica dei problemi e coloro che invece reclamano un approccio politico alle medesime questioni. Di conseguenza, la politicizzazione non è una caratteristica insita nella natura di un problema, ma spesso è il risultato di un tentativo di rompere le mura della cittadella tecnocratica.

4 Page (1997, 3-4) osserva che «nella struttura decisionale dell'Unione europea è molto difficile parlare di un qualunque soggetto - organismo, gruppo o individuo che detenga il potere, se il termine potere si riferisce a una capacità di determinare quali norme l'Unione dovrebbe introdurre o come si dovrebbero spendere i denari».

5 La tipologia proposta nella figura 1 costituisce soltanto un primo passo funzionale alla presentazione dei miei argomenti. Dovrebbe essere considerata uno strumento utile a sottolineare la necessità di differenziare, in seno all'Unione, fra tecnocrazia, politica burocratica, comunità epistemiche e politicizzazione. 


\begin{tabular}{l|ccc}
\hline \multirow{3}{*}{ Salienza } & & \multicolumn{2}{c}{ Incertezza } \\
\cline { 3 - 4 } & Bassa & Alta \\
& Alta & Politica burocratica & Logica tecnocratica \\
& & Politicizzazione & $\begin{array}{l}\text { Comunità epistemiche e } \\
\text { imprenditorialità di policy }\end{array}$ \\
\hline
\end{tabular}

FIG. 1. Come cambia la logica prevalente di scelta pubblica al variare di incertezza e salienza.

Ciò premesso, l'idea di fondo è quella di indicare come le varie combinazioni di incertezza e salienza possano facilitare l'emergere di un tipo di logica rispetto a un altro. La prima dimensione - salienza - evidenzia la differenza fra politiche opache, isolate dal dibattito di opinione pubblica, poco visibili nell'agenda politica e parlamentare, e policies la cui valenza politica risulta maggiormente esposta. Stiamo quindi parlando della differenza fra politiche pubbliche con pubblici e senza pubblici, una distinzione ben nota agli studiosi di policy analysis. La seconda dimensione è quella dell'incertezza. Maggiore incertezza significa più spazio per una logica di tipo problem-solving basata su modalità efficientistico-tecnocratiche oppure epistemiche.

La politica dell'expertise risulta assente nel quadrante in basso a sinistra, quello della politicizzazione. Quando una tematica pubblica diviene ad alta esposizione politica e la conoscenza (idee e informazioni) è disponibile a basso costo, la negoziazione politica fra attori auto-interessati (non fra attori epistemici interessati al «bene comune») procede senza troppi impedimenti. Come sostiene Moravcsik (1998) l'analisi della imprenditorialità sovra-nazionale e delle comunità epistemiche deve procedere dall'assunto che la conoscenza non sia disponibile a basso costo. Solo in queste condizioni gli imprenditori sovranazionali (per esempio la Commissione) e gli attori epistemici hanno un vantaggio comparato nella produzione e manipolazione delle idee e dell'informazione. In conclusione, il tipico modo (mode) decisionale dovrebbe essere quello politico.

Tuttavia, quando la conoscenza non circola a basso costo, imprenditori di policy e comunità epistemiche possono sfruttare il loro vantaggio comparato nell'accesso e nella manipolazione 
di idee, informazione, soluzioni. La logica epistemica vale infatti in condizioni di incertezza radicale su temi visibili (quadrante in basso a destra), cioè laddove gli interessi degli stati non possono essere definiti con precisione e solo le logiche interpretativo-epistemiche (e/o imprenditori di policy sovra-nazionali al centro di dense reti) - tramite processi di apprendimento possono mettere a fuoco soluzioni altrimenti inaccessibili. $\mathrm{Al}$ riguardo, non sorprende che la letteratura comparata sulle comunità epistemiche abbia trattato con particolare successo il caso delle politiche ambientali a livello internazionale, una tematica ad alta temperatura politica e genuina incertezza sulle soluzioni.

Cosa accade quando la visibilità politica di un problema è limitata e non esiste molta complessità? Queste sono condizioni che facilitano la competizione «dura» per il potere, non i processi di apprendimento e di policy enlightenment. Le organizzazioni si troveranno impegnate in una logica di politica burocratica, con classiche battaglie per conquistarsi spazi in arene funzionali ben delimitate. Per fare un esempio, nel quadrante in alto a sinistra stanno $\mathrm{i}$ casi in cui le varie Dg della Commissione competono per assicurarsi il controllo dell'agenda.

Che rimane allora alla logica tecnocratica? Sebbene la teoria macro-politica tecnocratica sia viziata dai problemi menzionati prima, l'idea di un «modo» tecnocratico può essere valida quando in aree funzionali a bassa salienza una logica da professionisti abbina un repertorio di soluzioni tecnicamente sofisticate (ma in ultima analisi note: non siamo al cospetto di incertezza radicale) a un repertorio di problemi. Nel quadrante in alto a destra la logica prevalente è quella di lasciar fare chi sa trovare i mezzi più efficienti rispetto a un fine che si presume dato. Concludendo sul punto. La logica tecnocratica esiste, ma vi sono altre manifestazioni della politica dell'expertise. Il passo successivo consiste allora nell'andare a controllare empiricamente come diversi processi di policy comunitari si collocano rispetto ai quattro quadranti della figura. A questo compito viene dedicato il prossimo paragrafo.

\section{Dalla tecnocrazia alla politicizzazione?}

Qualche indicazione dagli studi di caso. In questo contesto, desidero anzitutto presentare qualche traccia dell'evidenza contenuta in alcuni studi completati di recente (Radaelli 1997; 
1999; Harcourt e Radaelli 1999) ${ }^{6}$ che nella loro parte empirica coprono la negoziazione dell'Unione Economica e Monetaria, la tassazione diretta e la regolazione degli assetti proprietari nei media. Gli studi del caso non consentono generalizzazioni di tipo statistico, però si prestano bene ad essere sviluppati sul piano teorico (Yin 1994). In secondo luogo, vorrei trattare la prospettica cognitiva in un contesto teorico: cosa vuol dire che la conoscenza conta? E davvero il punto di vista cognitivo è in grado di spiegare il mutamento politico (policy change)?

La tecnocrazia è una sfida per tutti i tre casi esaminati, ma non è insuperabile. Tanto per cominciare, la politica dell'expertise non è confinata alla tecnocrazia. Le comunità epistemiche, ad esempio, sono state influenti nella progettazione della moneta unica (Verdun 1999), e la politica burocratica pervade il settore della disciplina della regolazione dei media, in relazione alla quale differenti $\mathrm{Dg}$ della Commissione sono in concorrenza per fissare l'agenda (Harcourt 1998). Ancora più importante, nei tre casi esaminati il punto di partenza è stato di tipo tecnocratico (con forti accentuazioni epistemiche nel caso della negoziazione delle disposizioni monetarie di Maastricht), ma col tempo un processo di politicizzazione ha in ultima analisi controbilanciato il potere dell'expertise. In tal senso i problemi di policy esaminati sono stati traghettati verso il quadrante della politicizzazione nella figura 1 .

Dei tre casi, è quello delle disposizioni in materia monetaria sottoscritte a Maastricht ad offrire l'esempio più lampante del potere delle idee economiche, degli esperti non elettivi e dei professionisti. Nel complesso, il potere della conoscenza nell'Unione Economica e Monetaria è catturato meglio dalla categoria di comunità epistemica, che da quella di politica burocratica o tecnocrazia. Le prove a sostegno di questa affermazione sono offerte dal potere del Comitato Delors - responsabile della stesura del programma per la moneta unica. Secondo Verdun (1999; vedi anche Cameron 1995 e Moravcsik 1998) questo comitato ha agito come una comunità epistemica. Credo tuttavia che, pur accettando la proposizione che questo caso si avvicina molto al tipo ideale della logica epistemica, si debba fare un'osservazione. Il Comitato Delors fu qualcosa di più di una rete

6 Il lettore interessato ad ulteriore evidenza empirica dovrebbe consultare questi due studi e la restante letteratura sulle politiche pubbliche dell'Unione citata in questo paragrafo. 
epistemica con un obiettivo comune in termini di policy, dato che era presieduto dal leader imprenditoriale della Commissione, Delors. Quest'ultimo fu il presidente di un'organizzazione burocratica con un suo obiettivo preciso, e non semplicemente un altro membro di un club epistemico volto ad orientare-illuminare (enlighten) il processo di policy! Quindi darei una valenza doppia al comitato Delors, sia come manifestazione della logica epistemica che come indicazione empirica della imprenditorialità sovra-nazionale.

Ancora più importante, possiamo collocare questo caso nel quadrante in basso a destra della figura 1? Non in modo definitivo, dato che - questa è la mia conclusione - il modo decisionale politico è stato in ultima analisi quello prevalente. Questa conclusione può essere chiarita riflettendo sulla differenza tra fattibilità tecnica e accettabilità politica (Dyson e Featherstone 1999). L'expertise (sub specie comunità epistemiche e imprenditorialità di Delors) deve essere tenuta in grande considerazione per quanto riguarda la realizzabilità tecnica della moneta unica. Questa è la sfera nella quale le preferenze dei banchieri centrali sono state incorporate nel trattato. Più in generale, l'intera dimensione cognitiva dell'Unione monetaria è stata pervasa da credenze condivise relative all'indipendenza della banca centrale e alla credibilità che $a$ ) hanno avuto origine dal fallimento delle politiche economiche keynesiane, $b$ ) sono state sostenute da una particolare versione del monetarismo, e $c$ ) hanno trovato nella politica monetaria tedesca un esempio reale e desiderabile da imitare (McNamara 1998). La dimensione ideativa dell'Unione monetaria è stata rafforzata dal ruolo giocato dal Comitato Delors. Questa dimensione dell'Unione monetaria (vale a dire, la sua fattibilità tecnica) rivela una forte influenza delle comunità epistemiche e dell'imprenditorialità sovra-nazionale. C'è però un'altra dimensione del processo di policy che ha portato alla moneta unica: la sua accettabilità sotto il profilo politico. A Maastricht, la dimensione cognitiva della politica è stata accompagnata da un'intensa attività strategica fatta di costruzione di assi politici privilegiati ${ }^{7}$, di opportunità politiche ${ }^{8}$, e

7 Si pensi, ad esempio, al rapporto fra i ministeri degli esteri di Germania e Francia. Un altro esempio è la costruzione di una coalizione di monetaristi-istituzionalisti e la rottura del fronte degli economisti (con la defezione della Spagna) dopo le decisioni prese ad Ashford Castle.

8 La riunificazione tedesca è stata probabilmente la più grande opportunità politi- 
persino di negoziati segreti ${ }^{9}$. È stata questa dimensione prevalentemente politica a definire i confini di ciò che era accettabile $\mathrm{e}$, in ultima istanza, a trasformare un insieme di idee in un trattato. Concludendo, i fattori politici hanno giocato il ruolo in definitiva più importante.

La fiscalità diretta illustra come la stessa Commissione abbia potuto superare una fase di stallo passando da una logica tecnocratica - assolutamente prevalente nelle proposte della Commissione fino al 1995 - ad una discussione più politica sugli svantaggi della concorrenza fiscale sregolata. I progressi compiuti di recente in questo settore possono essere attribuiti senza dubbio a un rinnovato interesse politico per il coordinamento fiscale. La Commissione ha messo da parte il precedente approccio cauto, tecnico e non-politico (in una parola: tecnocratico) alla questione dell'efficienza fiscale in Europa - approccio dettato dal Commissario Christiane Scrivener, in carica fino al 1995 - ed ha spinto con forza nella direzione di una discussione politica sulle conseguenze della competizione fiscale dannosa. L'idea forza della Commissione è che la concorrenza senza limiti per attirare il capitale induce a tassare maggiormente il fattore lavoro - con gravi conseguenze per l'occupazione oppure - ove si decidesse di non compensare le minori entrate da capitale con un maggiore carico fiscale sul lavoro - una riduzione inaccettabile del combustibile fiscale necessario alla macchina del welfare state. Come si vede, siamo al cospetto di un dilemma squisitamente politico: come impedire che la liberalizzazione dei movimenti di capitale danneggi l'economia sociale di mercato.

Abbandonata ai dibattiti tecnocratici sull'efficienza dei sistemi fiscali europei, la politica fiscale diretta dell'Unione non sarebbe mai andata oltre l'assunzione di misure di modestissima entità. L'aspetto più interessante di questo processo è che i burocrati stessi (vale a dire, la Commissione) hanno politicizzato

ca a sostegno del negoziato sull'Unione Monetaria a Maastricht. Un'altra opportunità era rappresentata dalla possibilità di imprigionare la Bundesbank nel processo di formazione della policy dell'Unione Monetaria attraverso la costituzione del Comitato Delors. $\mathrm{Si}$ vedano in proposito le accurate - anche se a tratti divergenti - analisi di Dyson e Featherstone (1999) e Moravcsik (1998).

9 Il governo inglese, ad esempio, non era stato reso edotto delle implicazioni strategiche dell'istituzione del Comitato Delors. Inoltre, l'istituzionalizzazione dei rapporti franco-tedeschi è stata rafforzata da una serie di incontri segreti tenuti durante la conferenza intergovernativa. 
l'issue. Quindi in questo secondo caso metterei l'accento sul passaggio dal quadrante tecnocratico della figura 1 a quello della politicizzazione.

La regolazione della proprietà nel settore dei media offre un altro esempio della politica dell'expertise, ma mostra anche quanto cresca la politicizzazione. L'Ue si è impegnata nella regolazione della proprietà in questo settore facendo leva sui tipici argomenti efficientistici dell'armonizzazione (la tesi della parità dei punti di partenza), considerazioni di carattere tecnico sul futuro dell'industria dei media, e la logica del mercato unico (Harcourt 1998). Una significativa preoccupazione della Commissione è stata quella di evitare di impegolarsi in più ampie riflessioni sugli aspetti legati alla democraticità e alle particolarità dei media rispetto al pluralismo e alle identità culturali. Eppure anche qua la politicizzazione sta facendo progressi. Il discorso anti-tecnocratico fa leva sulla questione del pluralismo (Humphreys 1996; Harcourt e Radaelli 1999) e i suoi più fervidi sostenitori sono il Parlamento europeo e la Dg X della Commissione.

Nel complesso, la regolazione della proprietà dei media non rientra tanto facilmente in una delle quattro categorie precedentemente proposte (tecnocrazia, politica burocratica, comunità epistemiche e politicizzazione). Un candidato da escludere sono le comunità epistemiche. I decisori non debbono affrontare una situazione nella quale hanno dei dubbi circa i loro stessi interessi e le comunità di esperti favoriscono la definizione delle preferenze. La politica burocratica, al contrario, è al lavoro in questa area di policy, come dimostra il conflitto interno alla Commissione. Se la Dg X guarda alla regolazione dei media attraverso il paradigma del pluralismo, la XV opta per l'ottica del mercato unico, e la XIII vorrebbe che i media rientrassero nel grandioso disegno di una società dell'informazione (Harcourt 1998). Per quanto riguarda il futuro, la crescente importanza della società dell'informazione nell'agenda della Commissione potrebbe indirizzare il processo verso la regolazione tecnocratica, sebbene la politicizzazione, una volta scattata, non cede facilmente il campo. Giudicando sulla base della situazione attuale, sosterrei che la regolazione della proprietà dei media contiene elementi sia di politica burocratica sia di politicizzazione, con l'ombra della tecnocrazia che si stende sul futuro.

In conclusione, $\mathrm{i}$ casi in esame presentano i prerequisiti ideali per lo sviluppo della tecnocrazia in termini di formazione delle politiche pubbliche regolative, assenza di un pubblico at- 


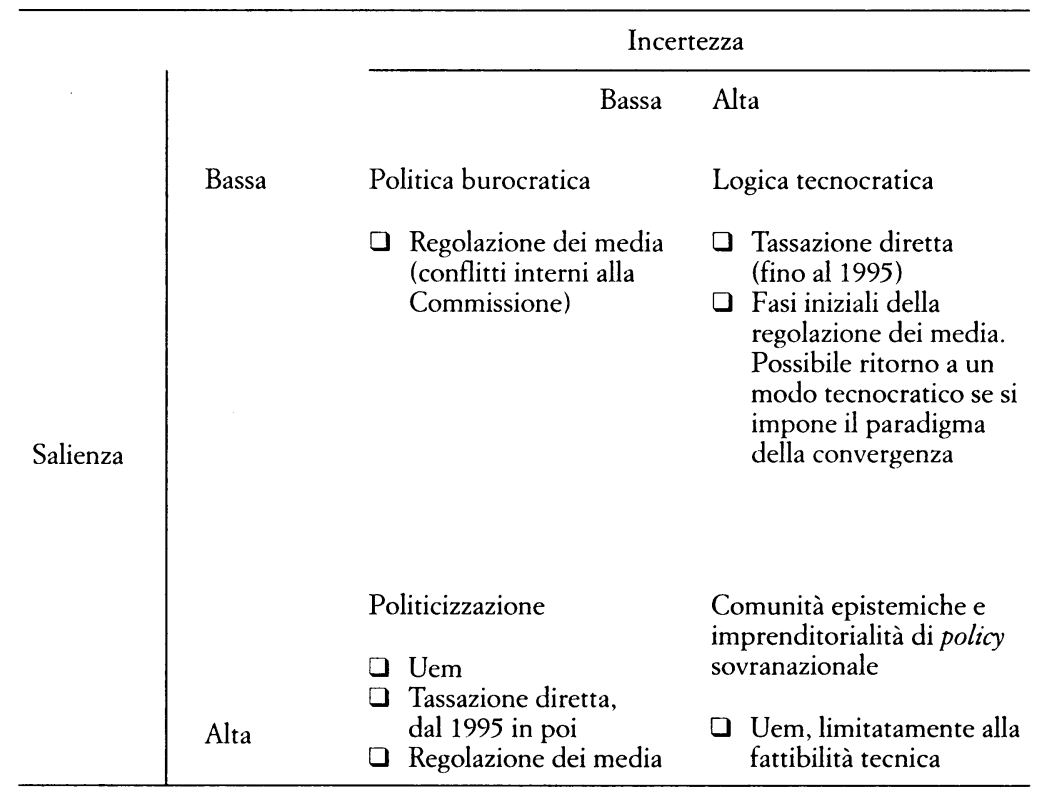

FIG. 2. La politicizzazione delle politiche europee.

tento, e il livello di expertise necessario a gestire queste questioni. Il potere dell'expertise è effettivamente elevato, ma non in condizione di annullare la tendenza generale verso la politicizzazione. La figura 2 riassume quanto abbiamo detto fino a questo punto.

La struttura della conoscenza. In una parola, «elevato» non significa «preponderante». Ma la dimensione cognitiva della politica non può certo essere trascurata soltanto perché non è preponderante. La sfida teorica, comunque, è costituita da come $\mathrm{i}$ modelli basati sulla conoscenza funzionano nella spiegazione del policy change (Capano 1995; Radaelli 1997). Nella trattazione di questo tema si andrà oltre gli approcci ritratti in figura 1 e si guarderà all'utilizzazione della conoscenza nel suo complesso, includendo anche l'approccio dell'advocacy coalition (Sabatier e Jenkins-Smith 1993). Codesto approccio richiama l'attenzione su un elemento fondamentale della strutturazione dei processi di policy, ovvero le coalizioni che fondono interessi, idee e conoscenza. Questo approccio propone anche una serie 
di proposizioni causali che possono essere verificate empiricamente. Tuttavia, l'approccio basato sulle coalizioni - quanto meno nella formulazione originale di Sabatier e Jenkins-Smith (1993), ma si veda anche Sabatier (1998) - non è completamente soddisfacente quando spiega il mutamento non incrementale come una conseguenza di modificazioni sostanziali dell'ambiente di policy. In ultima istanza, il cambiamento è infatti spiegato da Sabatier e Jenkins-Smith ricorrendo ad una variabile esogena: quando l'ambiente di policy muta, le risorse a disposizione degli attori ne sono modificate e ciò determina nuovi e diversi rapporti fra una coalizione e l'altra, laddove una coalizione riesca a sfruttare le modificazioni ambientali a suo vantaggio.

Il cambiamento può essere una conseguenza di shocks esogeni nell'ambiente di policy, ma questa proposizione sottovaluta quanto il sistema delle interazioni fra gli attori possa esso stesso produrre dinamica politica. L'interpretazione basata sugli elementi esogeni può essere giusta in molte circostanze, ma in certi casi necessita di essere integrata dalla riflessione su come l'apprendimento dentro il processo produca il cambiamento. L'ambiente di policy (ad esempio il mercato unico nel caso delle politiche per i media, la liberalizzazione dei movimenti di capitale in relazione alla politica fiscale, o le dinamiche delle politiche monetarie nazionali nel caso della moneta unica) gioca un ruolo importante nel delineare il contesto politico nell'ambito del quale le proposte per nuove iniziative dell'Unione diventano plausibili e necessarie. Inoltre, il mutamento di policy è facilitato dall'emergere di comunità epistemiche portatrici di paradigmi di policy.

Ciò non di meno, il mutamento ha luogo quando gli attori stessi divengono capaci di riflettere criticamente sulla loro interpretazione dei dilemmi che sorgono relativamente alle politiche pubbliche, e di conseguenza perseguono attivamente una diversa rappresentazione dei problemi. Di conseguenza l'apprendimento e la riflessione critica sulle interpretazioni (frame reflection) sono elementi endogeni che debbono essere accuratamente considerati. I modelli basati sull'interazione sociale (Lindblom 1990), il référentiel (Jobert 1989; Muller 1995) e le cornici concettuali (frames) (Schön e Rein 1994) hanno delle potenzialità rilevanti. Gli attori coinvolti in controversie sulle politiche pubbliche possono scoprire (e talvolta effettivamente scoprono), l'innovazione direttamente, attraverso un processo di apprendimento. A questo punto è possibile trarre una conclusio- 
ne: per guadagnare capacità esplicativa, le spiegazioni del policy change basate sulla conoscenza debbono includere tanto i fattori esogeni che quelli endogeni. Piuttosto che contrapporre gli elementi endogeni a quelli esogeni è utile sottolineare l'intreccio fra $\mathrm{i}$ due, che è poi l'intreccio fra interazione sociale, apprendimento e ambiente di policy.

Un altro argomento abbozzato in questo lavoro è il rifiuto di un punto di vista implicitamente presente in taluni filoni della ricerca, vale a dire la concezione «antropomorfica» della conoscenza. La tendenza a definire la conoscenza in rapporto a determinati attori (come esperti o burocrati) non convince completamente. Non è fruttuoso ipotizzare una corrispondenza biunivoca tra il ruolo politico della conoscenza e la partecipazione di attori particolari al processo di formazione delle politiche pubbliche. Al contrario, si potrebbe sostenere che la conoscenza ha meno a che fare con specifici attori che con la struttura nell'ambito della quale questi attori agiscono. Se la conoscenza conta, è perché essa si costituisce come struttura.

Vi è una somiglianza straordinaria fra la struttura del potere e quella della conoscenza. La struttura del potere è composta $\mathrm{da}$ istituzioni formali, rapporti di potere, regole decisionali, vincoli ed opportunità collegati alle possibilità di influenzare il comportamento. Gli elementi istituzionalizzati della struttura di potere sono dati per scontati dagli attori, ma esistono anche dinamiche evolutive. Allo stesso modo, la struttura cognitiva delle politiche pubbliche offre interpretazioni, rappresentazione di eventi politici, opportunità di apprendimento e «lenti» che consentono di mettere a fuoco interessi e codici di comportamento. A sua volta, l'apprendimento può essere un potente catalizzatore della modificazione della struttura cognitiva e, in ultima istanza, del policy change.

La struttura cognitiva comprende una conoscenza tecnica prodotta dalle scienze sociali, ma include anche una conoscenza interattiva, ovvero la conoscenza che un attore ha delle strategie degli altri attori. La conoscenza è prodotta - e non soltanto riprodotta - attraverso l'interazione sociale. Questo - per tornare ancora una volta sul dilemma endogeno versus esogeno - è il significato più profondo di endogeneità della conoscenza. Un corollario è che le preferenze non sono esogene nell'analisi politica. Le preferenze sono definite e modificate attraverso l'interazione sociale.

Il rapporto tra l'agire (agency) e la struttura è dialettico. In 
un dato momento, gli attori sono letteralmente immersi nella struttura cognitiva, ma quest'ultima esiste soltanto attraverso il comportamento degli attori. Per dirlo in modo diverso, la struttura deve essere agita (enacted) e interpretata (Giddens 1984; Wendt 1987, 359). Ciò ha implicazioni significative: in realtà, la componente cognitiva delle politiche pubbliche è riprodotta e istituzionalizzata (ovvero: acquisisce stabilità e influenza le politiche pubbliche stesse) soltanto se è riprodotta dai decisori pubblici i quali si adeguano a paradigmi interpretativi condivisi, restano attaccati alle opinioni comuni circa le realtà politiche, sociali ed economiche, e percepiscono il medesimo significato collegato alle stesse scelte pubbliche. $\mathrm{Ma}$ è proprio attraverso l'azione che gli attori contribuiscono allo sviluppo della struttura cognitiva, ad esempio modificando un'interpretazione via interazione sociale o scoprendo significati o esiti delle politiche pubbliche precedentemente non previsti. La struttura cognitiva è costante soltanto in una prospettiva statica. Ciò equivale a dire che in un dato momento la struttura cognitiva è data per scontata dagli attori perché è istituzionalizzata. Diventa però variabile quando l'analisi passa dalla staticità ad una prospettiva dinamica. In un dato momento l'istituzionalizzazione della conoscenza ha valore costitutivo rispetto all'azione in quanto attribuisce significato all'azione stessa. Ma «l'individuo (a differenza della cavia del comportamentista) traccia il percorso del labirinto mentre lo percorre» (Thompson, Ellis e Wildavsky 1990, 22).

La nozione giddensiana di «dualità della struttura» è, in questo caso, estremamente utile (Giddens 1984). La struttura è al tempo stesso il mezzo e il risultato non intenzionale delle pratiche sociali - sostiene Giddens. Gli attori ricorrono ad elementi della struttura (in particolare regole e risorse ${ }^{10}$ ) nell'esercizio (enactment) delle pratiche sociali, e utilizzando questi elementi allo stesso tempo concorrono alla riproduzione della struttura. Le strutture sociali non esistono indipendentemente dagli agenti, ma richiedono agenti per essere riprodotte. Come ha scritto Wendt, traendo spunto da Bhaskar e Giddens, «le strutture sociali hanno un'intrinseca dimensione discorsiva nel senso che sono inseparabili dalle ragioni e dai significati che gli attori attribuiscono alle loro azioni [...] Le strutture sociali sono ontologicamente dipendenti e di conseguenza costituite dalle pratiche e dalla percezione che gli attori hanno di se stessi»

10 In realtà Giddens considera tre elementi principali: struttura, risorse e moralità. 
(Wendt 1987, 359, corsivo nel testo). Di conseguenza, in qualsiasi fase del processo di riproduzione della struttura gli attori hanno la possibilità di introdurre cambiamenti.

Queste notazioni su struttura e azione vanno d'accordo con gli argomenti presentati in precedenza sulla necessità di integrare elementi endogeni ed esogeni. In conclusione, non è isolando e contrapponendo strutture esogene e azione endogena, ma piuttosto guardando all'interazione fra le due componenti, che le spiegazioni basate sulla conoscenza del mutamento delle politiche pubbliche possono guadagnare consistenza teorica.

\section{Considerazioni conclusive: integrare expertise e democrazia}

Come valutare la politica dell'expertise in seno all'Unione europea da un punto di vista normativo? Una raccomandazione che emerge da questo studio riguarda la necessità di integrare l'expertise nei progetti per un'Unione più democratica e legittimata. L'Ue è un sistema politico nell'ambito del quale l'expertise conta molto. La presenza di politica burocratica, comunità epistemiche, processi tecnocratici non è limitata all'Unione, ma la sua specializzazione nella produzione di politiche regolative accentua la spinosa questione dell'integrazione di expertise e democrazia. La soluzione individuata dai sistemi politici nazionali nei secoli passati - ossia, il rafforzamento del ruolo dei parlamenti nel controllo dell'esecutivo - non può essere considerata una panacea. Ovviamente, il Parlamento europeo ha giocato un ruolo di grandissima rilevanza nei recenti sviluppi. Nel caso delle politiche relative alla proprietà dei media, il Parlamento ha operato riportando il pluralismo fra i temi del dibattito. In quello delle politiche fiscali ha avallato la politicizzazione delle iniziative contro la concorrenza fiscale dannosa. Nel futuro, cercherà di far sentire la propria voce in relazione alle scelte di policy macroeconomiche. Una sorta di «coscienza dell'Unione Monetaria», insomma (Agence Europe, 31 ottobre 1998). Di conseguenza il Parlamento europeo ha un considerevole potenziale come agente della democratizzazione, specie se allo stesso tempo dovesse prender corpo un'identità europea (Schmitter 1999).

L'Unione europea deve comunque rafforzare la responsabilità e la capacità di risposta (responsiveness) della sua intera rete istituzionale. Rafforzare un giocatore a scapito degli altri po- 
trebbe risultare dannoso. Uno degli autori che ha studiato più a fondo la burocratizzazione del processo di formazione delle politiche pubbliche dell'Ue, Edward Page, ha concluso che «Accogliere più giocatori nel sistema, sia attraverso l'attribuzione di un ruolo più significativo al Parlamento europeo o ai legislativi nazionali o regionali e, forse ancora più importante, ai loro funzionari, potrebbe non ridurre il deficit democratico. Ampliare la gamma degli interessi e gli organismi che debbono essere armonizzati potrebbe rendere ancora più difficile identificare le responsabilità, trasformando un deficit democratico in un ancora meno democratico eccesso di istituzioni, gruppi ed individui che rivendicano tutti, con qualche valida ragione, di rappresentare i cittadini d'Europa» (Page 1997, 163).

Il controllo e la responsabilità democratica sono concetti in cerca di nuovi significati anche a livello nazionale. I parlamenti si trovano a dover fronteggiare sfide sempre più significative anche nell'ambito dei sistemi politici interni. La proliferazione di istituzioni non-maggioritarie (come le banche centrali indipendenti e le agenzie regolative) e di reti di attori politici non elettivi ha spinto autori quali Andersen e Burns (1996) a parlare di meccanismi di governo (governance) post-parlamentari. L'Ue costituisce un caso particolare di accentuazione di questa tendenza, date le fitte reti regolative e l'enfasi posta sull'efficienza (Dehousse 1997; Majone 1996). Questa delicata situazione richiede un salto in avanti, piuttosto che un viaggio a ritroso nel passato del controllo parlamentare.

In prospettiva, il crescente ruolo giocato dalla politicizzazione a scapito dell'expertise è indicativo di una positiva maturazione dell'Ue. La politica genera conflitto, struttura i cleavages, e polarizza l'opinione pubblica, ma allo stesso tempo libera l'Unione europea dalla trappola tecnocratica e apre nuovi spazi per un impiego più accettabile della politica dell'expertise. L'essenza della democrazia è il conflitto, e forse un certo tasso di inefficienza è il prezzo che si deve pagare per una più ampia partecipazione ed un dibattito più maturo, come dimostra il caso della regolazione della proprietà dei media (dove una proposta non è stata ancora presentata, sebbene la Commissione abbia pubblicato il suo primo Libro bianco sulla questione nel 1992). La politicizzazione può anche sbloccare l'impasse tecnocratico: la sfida della concorrenza fiscale può essere affrontata soltanto portando il discorso relativo sul piano della determinazione politica (come indica il codice di condotta Ue sulla con- 
correnza fiscale sregolata), e non ricorrendo a sterili approcci tecnocratici. E l'Euro non trasformerà l'Europa nel Paradiso grazie alle sue intrinseche virtù tecnocratiche. Al contrario, alla Banca Centrale Europea sarà chiesto di prendere decisioni politiche, scegliendo, ad esempio, se rispondere al problema della disoccupazione con un abbassamento dei tassi di interesse o meno.

La politicizzazione, in breve, contribuisce alla maturazione dell'Ue come comunità politica, e forse la renderà meno diversa di quanto non sia attualmente dai sistemi politici nazionali. Ciò non di meno, il problema di controllare le burocrazie regolative e gli esperti di policy non si risolverà automaticamente con il passare del tempo. Il problema non è se e quando $\mathrm{i}$ «mandarini d'Europa» saranno rimpiazzati dalla democrazia - non v'è dubbio che l'expertise sia destinata a giocare un ruolo anche in futuro -, ma se e come le politiche pubbliche europee potranno divenire più responsabili (accountable). La conclusione non è che la tecnocrazia sia scomparsa dall'Ue, ma che l'expertise (in varie forme: tecnocrazia, politica burocratica, comunità epistemiche e imprenditorialità di policy sovra-nazionale) opera in un ambiente sempre più politicizzato.

Il problema va oltre le procedure decisionali e investe il fondamento stesso dell'Unione. Vari autori hanno proposto diverse soluzioni (Andersen e Burns 1996; Majone 1996, cap. 13; O'Neill 1999; Schmitter 1999). Dalla particolare prospettiva scelta per questo studio, la principale raccomandazione è che le soluzioni tengano conto della specificità delle politiche pubbliche dell'Unione ${ }^{11}$. Politiche ad alto gradiente tecnico-cognitivo, da una parte, ed un contesto più politicizzato dall'altra, possono costituire sia una combinazione esplosiva sia un composto corroborante (se la minaccia tecnocratica viene trasformata in un contributo positivo dell'expertise). A questo proposito - $\mathrm{e}$ fermo restando che l'agenda della riforma include anche temi normativi fondamentali ma che esulano da questo studio - l'Ue dovrebbe guardare più ai casi di successo delle riforme regolative (Oecd 1997; Radaelli in corso di stampa) e a un affinamento del disegno istituzionale per l'Unione monetaria (Majone 1999)

11 Il Trattato di Amsterdam incarna la maturazione del dibattito, in quanto include norme che si ispirano al modello regolativo a fianco di norme basate sul modello parlamentare (Dehousse 1998). 
che al passato, ovvero, all'età dell'oro del controllo parlamentare. Una diffusa cultura della responsabilità, della legittimità procedurale $^{12}$, della credibilità dei regolatori dell'Unione, una sana competizione fra diversi organismi impegnati nella regolazione, il miglioramento della consultazione e il judicial review dovrebbero costituire macro-traiettorie (Majone 1996).

Al livello micro, degli strumenti, la qualità delle politiche pubbliche europee potrebbe giovarsi di una maggiore trasparenza dei criteri e delle scelte decisionali inerenti l'Euro, e di una possibile interazione fra governo economico (penso soprattutto a fiscalità "e occupazione) e governo monetario, ferma restando l'indipendenza della banca centrale. Le politiche regolative potrebbero giovarsi di analisi dell'impatto delle norme proposte, di regulatory budgets e del ricorso sistematico alla valutazione nell'ambito di una nuova cultura della democrazia in base ai risultati. Una Unione più responsabile è realizzabile, e il processo di politicizzazione potrebbe favorire, anziché indebolire, questo sviluppo. Se questa diventerà una realtà dipende dalle istituzioni europee, ma - come accennato - anche da processi assai più complessi, quali la priorità che i leader politici nazionali attribuiranno alle questioni della riforma dell'Unione, da modelli educativi e di socializzazione meno campanilistici e, in ultima istanza, dal decollo di una discussione attenta e consapevole. $\mathrm{Mi}$ auguro che la ricerca in questo settore possa contribuire allo sviluppo di tale consapevolezza.

\section{Riferimenti bibliografici}

Adler, E. e P.M. Haas (1992), Conclusion: epistemic communities, world order, and the creation of a reflective research program, in P.M. Haas (1992), pp. 367-390.

Allison, G.A. (1971), The Essence of Decision: Explaining the Cuban Missile Crisis, Boston, Little, Brown.

Andersen, S.S. e T. Burns (1996), The European Union and the erosion of parliamentary democracy: A study of post-parliamentary go-

12 Majone (1996, cap. 13) sostiene che la legittimità procedurale implica, fra le altre cose, procedure democratiche per la definizione degli obiettivi e del potere dei regolatori, e il requisito che costoro giustifichino le decisioni che hanno assunto. Quest'ultimo requisito - contenuto nell'art. 190 del Trattato di Roma - stimola la partecipazione pubblica, il controllo dei pari, e un ricorso sistematico all'analisi delle politiche pubbliche. Ciò rende le decisioni del regolatore soggette anche al controllo giudiziario. 
vernance in S.S. Andersen e K.A. Eliassen (a cura di), The European Union: How Democratic Is It? , London, Sage, pp. 227-251.

Axelrod, R. (1976), Structure of Decision. The Cognitive Maps of Political Elites, Princeton, Princeton University Press.

Boston, J. (1988), Advising the Prime Minister in New Zealand: the origins, functions and evolution of the Prime Minister's advisory group, in «Politics», n. 1, pp. 8-20.

Burris, B. (1993), Technocracy at Work, New York, State University of New York Press.

Cameron, D. (1995), Transnational Relations and the Development of European EMU, in T. Risse-Kappen (a cura di), Bringing Transnational Relations Back In, Cambridge, Cambridge University Press, pp. 37-78.

Capano, G. (1995), Il Policy change: tra politica delle idee e politica degli interessi, in «Teoria Politica», n. 1, pp. 133-165.

Caporaso, J. (1996), The European Union and Forms of State: Westphalian, Regulatory or Post-Modern?, in «Journal of Common Market Studies», n. 1, pp. 29-52.

Cini, M. (1996), La Commission Europenne: Lieu d'émergence de cultures administratives. L'exemple de la DG IV et de la DG XI, in «Revue Française de Science Politique», n. 3, pp. 457-472.

Coen, D. (1997), The evolution of the large firm as a political actor in the European Union, in «Journal of European Public Policy», n. 1, pp. 91-108.

Cram, L. (1993), Calling the tune without paying the piper? Social policy regulation: the role of the Commission in European Community social policy, in «Policy and Politics», n. 2, pp. 135-146.

Dehousse, R. (1997), Regulation by networks in the European Community: the role of European agencies, in «Journal of European $\mathrm{Pu}$ blic Policy», n. 2, pp. 246-261.

- (1998), European Institutional Architecture after Amsterdam: Parliamentary System or Regulatory Structure?, Working Paper n. 98/ 11, Firenze, Robert Schuman Centre, European University Institute.

Deutsch, K.W. (1966), The Nerves of Government, New York, The Free Press.

Downs, A. (1967), Inside Bureaucracy, Boston, Little, Brown.

Dyson, K. (1994), Elusive Union, Harlow, Longman.

Dyson, K. e K. Featherstone (1999), The Road to Maastricht. Negotiating the Maastricht Treaty, Oxford, Oxford University Press.

Featherstone, K. (1994), Jean Monnet and the Democratic Deficit in the EU, in «Journal of Common Market Studies», n. 2, pp. 149170.

Fischer, F. (1990), Technocracy and the Politics of Expertise, London, Sage. 
Fisichella, D. (1997), L'Altro Potere. Tecnocrazia e Gruppi di Pressione, Roma-Bari, Laterza.

Galbraith, J.K. (1967), The New Industrial State, Boston, Houghton Mifflin.

Giddens, A. (1984), The Constitution of Society: Outline of the Theory of Structuration, Cambridge, Polity Press.

Goldstein, J. (1993), Ideas, Interests, and American Trade Policy, Ithaca, Cornell University Press.

Giuliani, M. (1998), Sul concetto di imprenditore di policy, in «Rivista Italiana di Scienza Politica», n. 2, pp. 357-378.

Haas, E. (1958), The Uniting of Europe, Stanford, Stanford University Press.

Haas, P.M. (a cura di) (1992), Knowledge, Power and International Policy Coordination, in «International Organisation», Monographic Issue, n. 1.

Harcourt, A.J. (1998), EU media concentration. The conflict over the definition of alternatives, in «Journal of Common Market Studies», n. 3, pp. 369-389.

Harcourt, A.J. e C.M. Radaelli (1999), Limits to EU technocratic regulation?, in «European Journal of Political Research», n. 1, pp. 107-122.

Heclo, H. (1972), Review article: policy analysis, in «British Journal of Political Science», n. 2, pp. 83-108.

- (1974), Modern Social Politics in Britain and Sweden, New Haven, Yale University Press.

Humphreys, P. (1996), Media and Media Policy in Western Europe, Manchester, Manchester University Press.

Jacobsen, J.K. (1995), Much ado about ideas. The cognitive factor in economic policy, in «World Politics», pp. 283-310.

Jobert, B. (1989), The normative frameworks of public policy, in «Political Studies», pp. 376-386.

Lindblom, C.E. (1959), The science of muddling through, in «Public Administration», n. 1, pp. 78-88.

- (1990), Inquiry and Change. The Troubled Attempt to Understand and Shape Society, New Haven, Yale University Press.

Majone, G.D. (a cura di) (1996), Regulating Europe, London, Routledge.

- (1999), The regulatory state and its legitimacy problems, in «West European Politics», n. 1, pp. 1-24.

McNamara, K.R. (1998), The Currency of Ideas. Monetary Politics in the European Union, Ithaca-London, Cornell University Press.

Meynaud, J. (1969), Technocracy, New York, The Free Press.

Monnet, J. (1978), Memoirs, London, Collins.

Moravcsik, A. (1998), The Choice for Europe: Social Purpose and State Power from Messina to Maastricht, London, Ucl Press.

Muller, P. (1995), Les politiques publiques comme construction d'un 
rapport au monde, in A. Faure, G. Pollet, e P. Warin (a cura di), La Construction du Sens dans les Politiques Publiques. Débats autour de la Notion de Référentiel, Paris, L'Harmattan, pp. 153-179.

Oecd (1997), The Oecd Report on Regulatory Reform, Paris, Oecd Publications.

O'Neill, M. (1999), Between regime and republic: The polity problem in the European Union, Paper presented to the Uaces-Cespg-Amlrf workshop on The state of the art: theoretical approaches to the $E U$ in the post-Amsterdam era, Birmingham, Aston University, 6-7 maggio.

Page, E. (1997), People who Run Europe, Oxford, Oxford University Press.

Peters, G.B. (1992), Bureaucratic politics and the institutions of the European Community, in A. Sbragia (a cura di), Europolitics. Institutions and Policy-Making in the 'New' European Community, Washington, Brookings, pp. 75-122.

- (1994), Agenda-setting in the European Community, in «Journal of European Public Policy», n. 1, pp. 9-26.

Peterson, J. (1992), The European Technology Community Policy Networks in a Supranational Setting in D. Marsh e R.A.W. Rhodes (a cura di), Policy Networks in British Government, Oxford, Oxford University Press.

Putnam, R. (1977), Elite transformation in advanced industrial societies: an empirical assessment of the theory of technocracy, in «Comparative Political Studies», n. 3, pp. 383-412.

Radaelli, C.M. (1997), The Politics of Corporate Taxation in the European Union. Knowledge and International Policy Agendas, London, Routledge.

- (1999), Technocracy in the European Union, London, Longman.

- (in corso di stampa), Steering the EU regulatory system: the challenges abead, in «Public Administration», n. 4.

Richardson, J.J. (1995), Eu water policy. Uncertain agendas, shifting networks, and complex coalitions, in H. Bressers, L. O'Toole e J.J. Richardson (a cura di), Networks for water policy, Special issue di «Environmental Politics», pp. 139-167.

- (1996), Policy-making in the EU: ideas and garbage-cans of primeval soup, in J.J. Richardson (a cura di), The European Union: Power and Policy-Making, London, Routledge, pp. 3-23.

Rosen, S. (a cura di) (1973), Testing the Theory of the Military-Industrial Complex, Lexington (Mass.), Lexington Books.

Sabatier, P.A. e H.C. Jenkins-Smith (a cura di) (1993), Policy Change and Learning. An Advocacy Coalition Approach, Boulder, Westview.

Sabatier, P.A. (1998), The advocacy coalition framework: Revisions and relevance for Europe, in «Journal of European Public Policy», n. 1, pp. 98-130. 
Sartori, G. (1987), The Theory of Democracy Revisited, Chatham, Chatham House Publishers.

Schmitter, P. (1999), Come e perché democratizzare l'Unione europea, Bologna, Il Mulino.

Schön, D. e M. Rein (1994), Frame Reflection, New York, Basic Books.

Sclove, R.E. (1995), Democracy and Technology, New York, Guilford.

Simon, H. (1957), Models of Man, New York, Wiley.

Stone, D., A. Denham e M. Garnett (a cura di) (1998), Think Tanks across the World. A Comparative Perspective, Manchester, Manchester University Press.

Thompson, M., R. Ellis e A. Wildavski (1990), Cultural Theory, Boulder, Westview Press.

Verdun, A. (1999), The role of the Delors Committee in the creation of EMU: An epistemic community?, in «Journal of European Public Policy», n. 3, pp. 308-328.

Wallace, W. e J. Smith (1995), Democracy or technocracy? European integration and the problem of popular consent, in «West European Politics», n. 3, pp. 137-157.

Wallis, J. (1997), Conspiracy and the policy process: a case study of the New Zealand Experiment, in «Journal of Public Policy», n. 1, pp. 1-31.

Wendt, A.E. (1987), The agent-structure problem in international relations theory, in «International Organization», n. 3, pp. 441-473.

Williams, R. (1971), Politics and Technology, Basingstoke, Macmillan.

Wincott, D. (1998), Does the EU pervert democracy? Questions of democracy in new constitutionalist thought on the future of Europe, in «European Law Journal», n. 4, pp. 411-428.

Yin, R.K. (1994), Case Study Research, London, Sage, II ed.

Zito, A. (1998), Epistemic Communities and European Integration, Paper prepared for Ecpr Workshop n. 22 on The Role of Ideas in Policy-making, Ecpr $26^{\text {th }}$ Joint Sessions of Workshops, University of Warwick, 23-28 marzo. 